Originally published as:

Böhmer, M.M., Hellenbrand, W., Matysiak-Klose, D., Heininger, U., Müters, S., Wichmann, O. Pertussis vaccination coverage among adults in Germany [Pertussis-Impfquoten bei Erwachsenen in Deutschland] (2013) Deutsche Medizinische Wochenschrift, 138 (28-29), pp. 1451-1457.

DOI: $10.1055 / \mathrm{s}-0033-1343249$

This is an author manuscript.

The definitive version is available at: http://www.thieme-connect.de/ 


\section{Pertussis-Impfquoten bei Erwachsenen in Deutschland}

\section{Pertussis vaccination coverage among adults in Germany}

Merle M. Böhmer, ${ }^{\mathrm{a}, \mathrm{d}^{*}}$, Wiebke Hellenbrand ${ }^{\mathrm{a}}$, Dorothea Matysiak-Klose ${ }^{\mathrm{a}}$, Ulrich Heininger ${ }^{\mathrm{b}}$, Stephan Müters ${ }^{\mathrm{c}}$, Ole Wichmann ${ }^{\mathrm{a}}$

${ }^{a}$ Fachgebiet Impfprävention, Robert Koch-Institut, Berlin

${ }^{\mathrm{b}}$ Universitäts-Kinderspital beider Basel (UKBB), Basel, Schweiz

${ }^{\mathrm{c}}$ Abteilung für Epidemiologie und Gesundheitsberichterstattung, Robert Koch-Institut, Berlin

${ }^{\mathrm{d}}$ Charité - Universitätsmedizin Berlin, Berlin

* Korrespondenzautorin:

Merle M. Böhmer

Fachgebiet Impfprävention,

Abteilung für Infektionsepidemiologie,

Robert Koch-Institut,

DGZ-Ring 1, 13086 Berlin

E-Mail: BoehmerM@rki.de

Zeichenzahl (exklusive Leerzeichen)

Zusammenfassung (deutsch):1.646 Zeichen

Abstract (englisch):

1.372 Zeichen

Text:

30.282 (inkl. Tabellen, Abbildungen, Literaturverzeichnis)

Literaturstellen: $\quad \mathrm{n}=31(\max .40)$ 


\section{Zusammenfassung}

Hintergrund. Während in Deutschland seit 2001 eine Pertussis-Impfempfehlung für Risikogruppen (z.B. Personal im medizinischen Bereich/ in Gemeinschaftseinrichtungen, Kontaktpersonen von Säuglingen) besteht, wird erst seit 2009 von der Ständigen Impfkommission allen Erwachsenen die einmalige Pertussis-Impfung bei der nächsten fälligen Tetanus-Diphtherie-Auffrischimpfung empfohlen. Studienziele waren: 1) Erhebung von Pertussis-Impfquoten bei Erwachsenen, 2) Abschätzung der Verwendungshäufigkeit tetanushaltiger Kombinationsimpfstoffe in Krankenhäusern.

Methoden. Für 1) wurden Daten zweier bevölkerungsrepräsentativer Telefonsurveys unter Erwachsenen 2009/2010 analysiert (GEDA09: $n=21.262$; GEDA10: $\mathrm{n}=22.050$ ).

Einflussfaktoren des Pertussis-Impfstatus in Risikogruppen wurden mit logistischen Regressionsverfahren bestimmt. Für 2) wurde eine Befragung von 133 Krankenhausapotheken (betreuen 454 Krankenhäuser) für das Jahr 2007 durchgeführt. Ergebnisse. In GEDA10 hatten 5,9\% (95\%-Konfidenzintervall (KI): 5,5-6,3) der Befragten einen ausreichenden Pertussis-Impfschutz (Impfung in letzten 10 Jahren). Erwachsene, die einer Risikogruppe angehörten, hatten eine Impfquote von 10,7\% (95\%-KI: 9,8-11,7). Mit höheren Pertussis-Impfquoten in Risikogruppen signifikant assoziiert waren u.a. Wohnort in den Neuen Bundesländern und jüngeres Alter. Entgegen geltender Empfehlung wurden ca. $75 \%$ der Tetanus-Impfungen in Notaufnahmen bzw. Stationen als monovalente Impfung verabreicht.

Schlussfolgerungen. Angesichts hoher Pertussis-Inzidenzen und niedriger Durchimpfung in Risikogruppen und Allgemeinbevölkerung ist die Steigerung der Pertussis-Impfquoten in Deutschland dringend geboten. Impfquotensteigernde Maßnahmen (z.B.

Informationskampagnen) sollten unbedingt die impfende Ärzteschaft (Niedergelassene und Krankenhausärzte) miteinbeziehen.

Schlüsselwörter: Impfung, Impfquoten, Pertussis, Keuchhusten, Deutschland 
$\underline{\text { Background. }}$ Pertussis vaccination for risk-groups (e.g. healthcare workers, employees of communal facilities, or persons with close contact to infants) has been recommended in Germany since 2001. In 2009, single-dose acellular pertussis (ap) vaccination was recommended for all adults at the next tetanus-diphtheria (Td) booster. Study aims were to assess 1) pertussis vaccination coverage in adults, and 2) use of tetanus-containing combination vaccines in hospitals.

Methods. For 1) we analysed data from two population-based telephone surveys conducted among adults in Germany in 2009/2010 (GEDA09: $n=21,262$; GEDA10: $n=22,050$ ). Factors associated with vaccination were identified by logistic regression analyses. For 2) a questionnaire survey of 133 hospital pharmacies serving 454 German hospitals was undertaken for the year 2007.

Results. Overall, 5.9\% (95\% confidence interval (CI): 5.5-6.3\%) of GEDA10 participants reported up-to-date pertussis vaccination (ap-vaccination in past 10 years). In risk-groups, vaccination coverage was 10.7\% (95\%CI: 9.8-11.7\%). Residence in former East-Germany and younger age were independently associated with an adequate vaccination status. Contrary to prevailing recommendations, $\sim 75 \%$ of tetanus vaccines were administered as monovalent rather than Td- (or Tdap-)combination vaccines in hospitals.

Conclusions. In light of high pertussis-incidence and low vaccination coverage in German adults, improvement of pertussis vaccine uptake is vital, e.g. through awareness campaigns targeting both physicians in private practice and hospitals.

Keywords: vaccination, coverage, pertussis, whooping cough, Germany 


\section{Hintergrund}

Pertussis (Keuchhusten) ist eine hoch ansteckende respiratorische Infektionskrankheit, die durch das Bakterium Bordetella (B.) pertussis verursacht wird [25,29]. Der klinische Verlauf von Pertussis ist bei Säuglingen am schwersten und diese haben auch das höchste Risiko für Komplikationen, Hospitalisierung und Tod [8,10,24]. Obschon Pertussis bei älteren Kindern, Jugendlichen und Erwachsenen in der Regel weniger schwer verläuft als im Säuglingsalter, so ist die Krankheit oft protrahiert und Komplikationen wie Pneumonie, Harninkontinenz, Gewichtsverlust und Rippenfrakturen sind beschrieben [7,17,23]. Zudem kann es zur Übertragung der Erreger von erkrankten Erwachsenen auf ungeschützte Säuglinge kommen [26]. Weder die Infektion noch die Impfung gegen Pertussis führen zu einer lebenslangen Immunität. Nach natürlicher Infektion wird die Immunitätsdauer auf 4 bis 20 Jahre und nach Impfung auf 4 bis 12 Jahre geschätzt [27]. Ähnliche klinische Verläufe, jedoch zumeist leichteren Schweregrades, können durch B. parapertussis Infektion hervorgerufen werden, vor der die verfügbaren Pertussis-Impfstoffe nicht sicher schützen [11,31].

Die Krankheitslast durch Pertussis in Deutschland ist nach wie vor beträchtlich, mit einer Gesamtinzidenz von 27-40 Fällen/100.000 Einwohner in den fünf Neuen Bundesländern (NBL: Brandenburg, Mecklenburg-Vorpommern, Sachsen, Sachsen-Anhalt, Thüringen) in den Jahren 2008-2011 [21]. Erwachsene im Alter von $\geq 20$ Jahren machten 70\% aller Fälle aus. Bei Säuglingen zeigen Daten der Krankenhausdiagnosestatistik die höchste Krankheitslast (56 bzw. 23 Hospitalisierungen/100.000 Säuglinge im Jahr 2009 in den alten bzw. neuen Bundesländern) [21].

Die Ständige Impfkommission (STIKO) am Robert Koch-Institut (RKI) empfiehlt seit Juli 2009 aufgrund der beständig hohen Krankheitslast, auch bei Erwachsenen, eine einmalige Impfung gegen Pertussis generell für alle Erwachsenen [20]. In Ermangelung eines Einzelimpfstoffs soll die Pertussis-Impfung bei der nächsten fälligen Tetanus-Diphtherie (Td)-Auffrischimpfung durch die Verabreichung eines Tdap-Kombinationsimpfstoffes erfolgen [20]. Die Empfehlungen zur Impfung gegen Pertussis im Erwachsenenalter gelten auch im Rahmen einer Tetanusprophylaxe im Verletzungsfall. Eine berufliche Indikation besteht bereits seit 2001 für Personen, die in der Pädiatrie, der Infektionsmedizin oder in Gemeinschaftseinrichtungen tätig sind [18]. Diese Indikation wurde mehrmals erweitert, zuletzt im Jahr 2009 auf das gesamte Personal im Gesundheitsdienst [20]. Wegen eines zunehmenden Anteils von Pertussisfällen bei Erwachsenen empfahl die STIKO 2004 darüber hinaus eine Impfung von erwachsenen Personen mit engem Kontakt zu Säuglingen, 
einschließlich aller Frauen im gebärfähigen Alter, sofern in den letzten 10 Jahren keine Pertussis-Impfung stattgefunden hatte [19].

In Ermangelung eines nationalen Impfregisters lagen für Deutschland bislang keine Daten zur Umsetzung der seit mehreren Jahren bestehenden Empfehlung zur PertussisImpfung für Risikogruppen vor. Auch fehlten bisher Daten, die als Ausgangswert für die zukünftige Evaluation der Umsetzung der seit Juli 2009 bestehenden allgemeinen PertussisImpfempfehlung für Erwachsene dienen können. Um diese Datenlücke zu schließen wurden Daten zum Pertussis-Impfstatus im Rahmen der Studie „Gesundheit in Deutschland aktuell“ (GEDA) erhoben.

Es wird angenommen, dass ein beträchtlicher Anteil der Tetanusimpfungen (und damit potentiell auch Pertussis-Impfungen) bei Erwachsenen in Krankenhäusern im Rahmen der Tetanusprophylaxe im Verletzungsfall durchgeführt wird. Zusätzlich zu den GEDA-Daten werden hier daher die Ergebnisse einer Studie zur Compliance mit der Empfehlung, auch im Verletzungsfall mit tetanushaltigen Kombinationsimpfstoffen zu impfen, vorgestellt. Dazu wurde 2008 in Vorbereitung auf die allgemeine Pertussis-Impfempfehlung für Erwachsene eine Befragung von Krankenhausapotheken durchgeführt. Ziel dieser Befragung war es, für das Jahr 2007 zu erfassen, ob in Krankenhäusern - wie zum damaligen Zeitpunkt empfohlen - Td-Kombinationsimpfstoffe (im Gegensatz zu monovalenten Tetanusimpfstoffen (T)) eingesetzt wurden. Die Compliance mit der damaligen Empfehlung zu Td-Kombinationsimpfstoffen würde - so die Annahme - auch Rückschlüsse auf die zu erwartende Compliance mit der Empfehlung zur Verwendung von TdapKombinationsimpfstoffen zulassen. 


\section{Methoden}

\section{GEDA}

Zur Bestimmung der Pertussis-Impfquoten in Risikogruppen und der Allgemeinbevölkerung bei Erwachsenen in Deutschland wurden Daten der Studie ,Gesundheit in Deutschland aktuell' (GEDA) herangezogen. Im Rahmen dieser Studie zu Pertussis wurden Daten der ersten beiden GEDA-Surveys (GEDA09, GEDA10) ausgewertet. Einzelheiten zum Studiendesign von GEDA sowie zu den Charakteristika der Studienpopulationen von GEDA09 und GEDA10 wurden bereits beschrieben [3,4,22]. Bei GEDA handelt es sich um eine computergestützte telefonische Befragung Erwachsener zu gesundheitsrelevanten Themen, die im Rahmen des nationalen Gesundheitsmonitorings vom RKI durchgeführt wird [13]. Die Studienpopulation ist repräsentativ für die volljährige deutschsprachige Wohnbevölkerung, die in Privathaushalten in Deutschland lebt und über Festnetzanschlüsse erreichbar ist. Insgesamt wurden 21.262 Personen $\geq 18$ Jahre für GEDA09 und 22.050 Personen für GEDA10 interviewt. Der Altersmedian lag sowohl bei GEDA09 als auch bei GEDA10 bei 48,0 Jahren (Spannbreite: 18-100 Jahre bzw. 18-99 Jahre). Um einer systematischen Verzerrung z.B. durch Nonresponse vorzubeugen und somit die Repräsentativität der in dieser Studie getroffenen Aussagen zu gewährleisten, wurden alle Analysen mit einem Gewichtungsfaktor durchgeführt, der die Netto-Stichprobe an die Bevölkerungsstruktur hinsichtlich Alter, Geschlecht, Region und Bildungsstatus angleicht. Bei der im Ergebnisteil berichteten Responserate für GEDA09 und GEDA10 handelt es sich um die von der American Association of Public Opinion Research (AAPOR) definierte ,Response Rate 3' [1]. Response Rate 3 gibt den Anteil der realisierten Interviews im Verhältnis zu allen wahrscheinlichen Haushalten der Grundgesamtheit wieder. Für diejenigen Telefonnummern, bei denen zum Ende der Feldphase unklar blieb, ob sie Elemente der Grundgesamtheit sind oder nicht, wird dabei eine Schätzung vorgenommen, wie viele von diesen der Grundgesamtheit angehören. Diese Schätzung basiert auf den erhobenen Daten, und zwar als Anteil der Haushalte der Grundgesamtheit an allen Nummern, für die der Status, ob sie der Grundgesamtheit angehören oder nicht, festgestellt werden konnte. Für GEDA09 lag die Responserate bei $29,1 \%$, für GEDA10 bei $28,9 \%$. 
Zur Bestimmung der Pertussis-Impfquoten wurden Studienteilnehmer sowohl in GEDA09 als auch in GEDA10 gefragt, ob sie sich jemals gegen Pertussis haben impfen lassen und ob die letzte Pertussis-Impfung mehr oder weniger als 10 Jahre zurückliegt. Alle Befragten, die angaben, entsprechend den STIKO-Empfehlungen [12] in den letzten 10 Jahren eine Pertussis-Impfung erhalten zu haben, wurden als ausreichend geimpft gewertet. Studienteilnehmer/innen, die angegeben hatten, in einer Gemeinschaftseinrichtung oder in einem medizinischen Beruf tätig zu sein, mit Kindern $<1$ Jahr im Haushalt zu leben oder zur Gruppe der ,gebärfähigen Frauen` (Frauen $\leq 49$ Jahre) zu gehören, wurden als Risikogruppe für Pertussis eingestuft. Unterschiedliche Stufen des sozioökonomischen Status wurden, wie von Lampert et al. beschrieben [14], auf der Basis von Bildungs-, Einkommens- und beruflichem Status der Studienteilnehmer gebildet. Berlin wurde den NBL zugeordnet. Ein ,beidseitiger Migrationshintergrund“ lag vor, wenn der/die Studienteilnehmer/in selbst oder beide Elternteile nicht in Deutschland geboren wurden, ein , einseitiger Migrationshintergrund“ wenn ein Elternteil, beziehungsweise ,kein Migrationshintergrund“ wenn beide Elternteile in Deutschland geboren wurden.

Die statistischen Analysen wurden mit dem Software-Paket PASW 18.0 (SPSS Inc., Chicago, USA) unter Anwendung der Analysemethoden für komplexe Stichproben durchgeführt. Kategoriale Variablen wurden als Häufigkeiten (in Prozent) dargestellt. Soweit erforderlich, wurden darüber hinaus 95\%-Konfidenzintervalle (KI) sowie Odds Ratios (OR; Quotenverhältnisse) angegeben. Bivariate Assoziationen von kategorialen Variablen wurden mit Hilfe des Chi-Quadrat-Tests nach Pearson berechnet. Unterschiede zwischen Altersgruppen wurden für die binäre Variable zum Pertussisimpfstatus mittels des CochranArmitage-Test für Trends geprüft. Unabhängige Einflussfaktoren des Pertussis-Impfstatus wurden mit Hilfe von logistischen Regressionen eruiert. Ein $\mathrm{p}$-Wert $<0,05$ wurde als statistisch signifikant angenommen. Soweit nicht anders angegeben, handelt es sich bei allen nachfolgend dargestellten Zahlen um gewichtete Angaben.

\section{Krankenhausapotheken-Survey}

Um einen Einblick in die Impfpraktiken in Notaufnahmen zu erhalten, wurden 2008 in Zusammenarbeit mit dem Bundesverband Deutscher Krankenhausapotheker (ADKA) Krankenhausapotheken zur Anwendung tetanushaltiger Impfstoffe in Krankenhäusern im Jahr 2007 befragt. Von ca. 1.700 Krankenhausapothekern, die zum Zeitpunkt der Studie in 450 Krankenhausapotheken in Deutschland arbeiteten, waren 1.415 Mitglied im ADKA. Von 
diesen konnten 950 über einen internen Mail-Verteiler des ADKA angeschrieben und um Beantwortung von Fragen zu folgenden Faktoren gebeten werden: Anzahl und Art der belieferten Krankenhäuser (Erwachsenen-/pädiatrische, Notfallversorgung), Vorhaltung der verschiedenen tetanushaltigen Impfstoffe (Tetanus monovalent $(T)$, Tetanus-Diphtherie (Td), Tetanus-Diphtherie zusammen mit azellulärem Pertussisbestandteil für <5-Jährige (TDaP) und für >4-Jährige (Tdap)). Zudem wurde nach der Anzahl der abgegeben Dosen des jeweiligen Impfstoffes an Stationen bzw. an Notaufnahmen gefragt. 


\section{Ergebnisse}

\section{$\underline{\text { GEDA }}$}

Informationen zum Pertussis-Impfschutz lagen für 68,3\% (95\% KI: 67,5-69,1) der Studienteilnehmer/innen von GEDA09 und für 71,4\% (95\% KI: 70,7-72,2) der Teilnehmer/innen von GEDA10 vor. Weitere 31,5\% (95\% KI: 30,7-32,4) bzw. 28,3\% (95\% KI: 27,5-29,0) gaben an, nicht zu wissen, ob sie in den letzten 10 Jahren gegen Pertussis geimpft worden sind. Die Analyse der Teilnehmer/innen, die in GEDA10 ,weiß nicht' angegeben hatten, ergab in Bezug auf die Variablen Geschlecht (männlich/weiblich), Wohnort (ABL/NBL) und Altersgruppe (18-39/40-59/ 260 Jahre) ähnliche Verteilungen wie die Gruppe der Nicht-Geimpften.

Eine Pertussis-Impfung in den letzten 10 Jahren hatten 5,1\% bzw. 5,9\% erhalten (Tab.1). Der beobachtete Anstieg von 0,8 Prozentpunkten zwischen den beiden GEDABefragungen war nicht signifikant. Weitere Impfquoten für Pertussis nach Geschlecht, Alter, soziodemographischen Parametern sowie verschiedenen Indikationsstellungen für eine Pertussis-Impfung sind in Tabelle 1 dargestellt. Der Anteil von Personen mit ausreichendem Pertussis-Impfschutz nahm mit zunehmendem Alter ab (Abb. 1; Test für Trend für beide Studienpopulationen jeweils $\mathrm{p}<0,001$ ). 


\section{Tabelle 1}

Pertussis-Impfquoten (Impfung innerhalb der letzten 10 Jahre) bei Erwachsenen in Deutschland nach Geschlecht, Wohnort, Alter, Risikogruppe und weiteren Parametern

\begin{tabular}{|c|c|c|c|c|c|c|}
\hline & \multicolumn{3}{|c|}{ GEDA09 } & \multicolumn{3}{|c|}{ GEDA10 } \\
\hline & \multicolumn{2}{|c|}{ Fallbasis (ungewichtet), n } & \multirow{2}{*}{$\begin{array}{c}\text { Impfquote } \\
\%(95 \% \mathrm{KI})^{\#}\end{array}$} & \multicolumn{2}{|c|}{ Fallbasis (ungewichtet), n } & \multirow{2}{*}{$\begin{array}{c}\text { Impfquote } \\
\%(95 \% \mathrm{KI})^{\#}\end{array}$} \\
\hline & gesamt & $\begin{array}{l}\text { davon geimpft } \\
\text { gegen Pertussis }\end{array}$ & & gesamt & $\begin{array}{l}\text { davon geimpft } \\
\text { gegen Pertussis }\end{array}$ & \\
\hline Gesamt & 14.516 & 744 & $5,1(4,7-5,6)$ & 15.754 & 927 & $5,9(5,5-6,3)$ \\
\hline \multicolumn{7}{|c|}{ Soziodemographische Merkmale } \\
\hline \multicolumn{7}{|l|}{ Geschlecht } \\
\hline Männlich & 6.868 & 274 & $4,0(3,4-4,6)$ & 7.448 & 355 & $4,8(4,2-5,4)$ \\
\hline Weiblich & 7.648 & 470 & $6,1(5,6-6,8)^{* *}$ & 8.306 & 572 & $6,9(6,3-7,5)^{* * *}$ \\
\hline \multicolumn{7}{|l|}{ Wohnort } \\
\hline $\mathrm{ABL}$ & 11.568 & 420 & $4,3(3,8-4,9)$ & 12.478 & 553 & $4,4(4,1-4,9)$ \\
\hline NBL & 2.948 & 323 & $13,1(11,1-15,2)^{* *}$ & 3.276 & 373 & $11,4(10,1-12,8)^{* *}$ \\
\hline \multicolumn{7}{|l|}{ Altersgruppe } \\
\hline 18-39 Jahre & 4.604 & 458 & $10,0(9,0-11,0)$ & 4.855 & 523 & $10,8(9,8-11,8)$ \\
\hline 40-59 Jahre & 5.472 & 187 & $3,4(2,9-4,0)^{* *}$ & 5.958 & 314 & $5,3(4,6-6,0)^{* *}$ \\
\hline$\geq 60$ Jahre & 4.440 & 99 & $2,2(1,6-3,0)^{* *}$ & 4.941 & 90 & $1,8(1,4-2,3)^{* *}$ \\
\hline \multicolumn{7}{|c|}{ Sozioökonomischer Status } \\
\hline Niedrig & 2.853 & 134 & $4,5(3,5-5,7)$ & 3.255 & 134 & $4,1(3,3-5,1)$ \\
\hline Mittel & 8.587 & 453 & $5,3(4,7-5,9)$ & 9.323 & 578 & $6,2(5,6-6,8)^{* *}$ \\
\hline Hoch & 2.879 & 152 & $5,3(4,6-6,1)$ & 3.126 & 213 & $6,8(6,0-7,7)^{* *}$ \\
\hline \multicolumn{7}{|c|}{ Migrationshintergrund } \\
\hline Beidseitig & 2.011 & 56 & $2,7(2,0-3,6)$ & 2.275 & 92 & $4,1(3,2-5,2)$ \\
\hline Einseitig & 464 & 17 & $3,6(2,3-5,5)$ & 552 & 32 & $5,8(4,0-8,4)$ \\
\hline Ohne & 11.983 & 672 & $5,6(5,1-6,1)^{* *}$ & 12.925 & 803 & $6,2(5,7-6,7)^{* *}$ \\
\hline \multicolumn{7}{|c|}{ Pertussis-Risikogruppen } \\
\hline \multicolumn{7}{|c|}{$\begin{array}{l}\text { Zugehörigkeit zu einer der } \\
\text { Risikogruppen für Pertussis }\end{array}$} \\
\hline Nein & 10.123 & 347 & $3,4(3,0-3,9)$ & 11.042 & 422 & $3,8(3,4-4,3)$ \\
\hline
\end{tabular}




\begin{tabular}{|c|c|c|c|c|c|c|}
\hline $\mathrm{Ja}$ & 4.393 & 397 & $9,0(8,2-10,0)^{* *}$ & 4.712 & 505 & $10,7(9,8-11,7)^{* *}$ \\
\hline \multicolumn{7}{|c|}{$\begin{array}{l}\text { Zugehörigkeit zur Gruppe } \\
\text {,Medizinisches Personal }\end{array}$} \\
\hline Nein & 13.804 & 647 & $4,7(4,3-5,1)$ & 14.828 & 780 & $5,3(4,9-5,7)$ \\
\hline $\mathrm{Ja}$ & 712 & 97 & $13,7(11,4-16,3)^{* *}$ & 926 & 147 & $16,4(13,9-19,3)^{* * *}$ \\
\hline \multicolumn{7}{|c|}{$\begin{array}{l}\text { Zugehörigkeit zur Gruppe } \\
\text {,Personal in Gemeinschaftseinrichtungen' }\end{array}$} \\
\hline Nein & 14.015 & 705 & $5,0(4,6-5,5)$ & 15.216 & 873 & $5,7(5,3-6,2)$ \\
\hline $\mathrm{Ja}$ & 501 & 38 & $7,7(5,7-10,2)^{*}$ & 538 & 54 & $10,1(7,7-13,0)^{* * *}$ \\
\hline \multicolumn{7}{|c|}{$\begin{array}{l}\text { Zugehörigkeit zur Gruppe } \\
\text {,Frauen im gebärfähigen Alter' ( } \leq 49 \text { Jahre) }\end{array}$} \\
\hline Nein & 10.701 & 385 & $3,6(3,2-4,1)$ & 11.718 & 480 & $4,1(3,7-4,6)$ \\
\hline $\mathrm{Ja}$ & 3.815 & 359 & $9,4(8,5-10,4)^{* *}$ & 4.036 & 447 & $11,1(10,1-12,2)^{* *}$ \\
\hline \multicolumn{7}{|c|}{ Kinder <1 Jahr im Haushalt lebend } \\
\hline Nein & 14.244 & 710 & $5,0(4,6-6,1)$ & 15.453 & 896 & $5,8(5,4-6,3)$ \\
\hline $\mathrm{Ja}$ & 272 & 34 & $12,5(8,3-18,5)^{* *}$ & 301 & 30 & $10,1(7,2-14,1)^{*}$ \\
\hline \multicolumn{7}{|c|}{ Sonstige Merkmale } \\
\hline \multicolumn{7}{|c|}{$\begin{array}{l}\text { Tetanusschutzimpfung in den letzten } \\
10 \text { Jahren erhalten }\end{array}$} \\
\hline Nein & 3.914 & 29 & $0,4(0,3-0,7)$ & 4.518 & 20 & $0,7(0,5-1,1)$ \\
\hline $\mathrm{Ja}$ & 10.131 & 699 & $8,3(7,7-8,9)^{* *}$ & 10.829 & 900 & $6,9(6,3-7,5)^{* *}$ \\
\hline
\end{tabular}

${ }^{\#}$ gewichtete Angaben; *p-Wert $<0,05 ; * *$ p-Wert $<0,001 ; \mathrm{KI}=$ Konfidenzintervall; $\mathrm{ABL}=$ Alte Bundesländer; NBL=Neue Bundesländer; ${ }^{1)}$ Risikogruppe definiert als Zugehörigkeit zu mindestens einer der folgenden Gruppen Medizinisches Personal, Personal in Gemeinschaftseinrichtungen, Frauen im gebärfähigen Alter, Personen mit Kinder $<1$ Jahr im Haushalt lebend; ${ }^{2}$ bis 2005 war auch eine monovalente Impfung gegen Pertussis möglich, danach nur noch als Kombinationsimpfung mit Diphtherie- und Tetanuskomponente 


\section{Abbildung 1}

Pertussis-Impfquoten bei Erwachsenen in Deutschland 2008/09 (GEDA09) und 2009/10 (GEDA10), nach Altersgruppen

Insgesamt hatten 30,0\% (95\% KI: 29,3-30,7) der Studienteilnehmer/innen von GEDA10 eine berufliche (medizinisches Personal, Personal in Gemeinschaftseinrichtungen) oder sonstige Indikation (Frauen im gebärfähigen Alter, Haushaltskontakte von Säuglingen) für eine Pertussis-Impfung. Für diese Risikogruppen zugehörigen Personen bestand dementsprechend seit längerem eine Empfehlung zur Indikationsimpfung gegen Pertussis. Für 71.4\% der Risikogruppe lagen Informationen zum Pertussis-Impfstatus vor. Einen ausreichenden Pertussis-Impfschutz hatten lediglich 10,7\% (95\% KI: 9,8-11,7). Weitere Impfquoten für zu Risikogruppen gehörende Erwachsene nach soziodemographischen Parametern sind in Tabelle 2 zu finden. Ebenfalls in Tabelle 2 befinden sich die Ergebnisse der univariaten und multivariablen Analyse von Faktoren, die potentiell mit dem Erhalt einer Pertussis-Impfung in den letzten 10 Jahren in Risikogruppen assoziiert sind. Personen aus den NBL, Jüngere (18-39 Jahre) und Personen, die innerhalb der letzten 10 Jahre eine TetanusImpfung erhalten haben, waren in der multivariablen Analyse signifikant häufiger geimpft im Vergleich zu Personen aus den ABL bzw. älteren Personen (ab 40 Jahre) bzw. Personen ohne Tetanus-Impfung in den letzten 10 Jahren. In einer entsprechenden Analyse der GEDA09Daten wurden die gleichen Faktoren wie die in Tabelle 2 dargestellten identifiziert. Zusätzlich zu diesen Faktoren waren jedoch in GEDA09 Personen ohne Migrationshintergrund signifikant häufiger geimpft als Personen mit beidseitigem Migrationshintergrund.

\section{Tabelle 2}

Univariate und multivariable Analyse von Faktoren, die potentiell mit dem Erhalt einer Pertussis-Impfung in den letzten 10 Jahren bei zu Risikogruppen gehörenden Erwachsenen assoziiert sind (Datenquelle: GEDA10; Fallbasis ungewichtet: $n=6.616$ )

\begin{tabular}{|c|c|c|c|}
\hline & \multirow[b]{2}{*}{$\begin{array}{c}\text { Impfquote } \\
\%(95 \% \mathrm{KI})^{\#}\end{array}$} & \multicolumn{2}{|c|}{ Odds Ratio $(95 \% \mathrm{KI})^{\#}$} \\
\hline & & Univariat & Multivariabel \\
\hline \multicolumn{4}{|l|}{ Geschlecht } \\
\hline Männlich & $9,6(6,8-13,4)$ & $1^{\text {Ref }}$ & n.s. \\
\hline Weiblich & $10,8(9,9-11,9)$ & $1,14(0,82-1,60)$ & \\
\hline \multicolumn{4}{|l|}{ Wohnort } \\
\hline $\mathrm{ABL}$ & $8,6(7,8-9,6)$ & $1^{\text {Ref }}$ & $1^{\text {Ref }}$ \\
\hline NBL & $19,2(16,4-22,4)$ & $2,52(2,06-3,07)^{* *}$ & $2,39(1,94-2,93)^{* *}$ \\
\hline \multicolumn{4}{|l|}{ Altersgruppe } \\
\hline 18-39 Jahre & $13,0(11,6-14,4)$ & $1^{\text {Ref }}$ & $1^{\text {Ref }}$ \\
\hline 40-59 Jahre & $7,9(6,7-9,2)$ & $0,58(0,47-0,70)^{* *}$ & $0,55(0,45-0,67)^{* *}$ \\
\hline$\geq 60$ Jahre & $7,1(3,4-14,3)$ & $0,51(0,21-1,28)$ & $0,59(0,23-1,52)$ \\
\hline
\end{tabular}




\begin{tabular}{|l|r|r|r|}
\hline \multicolumn{3}{|l|}{ Sozioökonomischer Status } & n.s. \\
\hline Niedrig & $8,9(6,7-11,7)$ & $1^{\text {Ref }}$ & \\
\hline Mittel & $11,0(9,8-12,4)$ & $1,27(0,96-1,68)$ & n.s. \\
\hline Hoch & $11,2(9,7-13,0)$ & $1,30(0,95-1,78)$ & \\
\hline Migrationshintergrund & $1^{\text {Ref }}$ & \\
\hline Beidseitig & $6,2(4,5-8,4)$ & $1,36(0,78-2,39)$ & $1^{\text {Ref }}$ \\
\hline Einseitig & $8,2(5,2-12,9)$ & $2,06(1,53-2,77)^{* *}$ & \\
\hline Ohne & $11,9(10,9-13,1)$ & $1^{\text {Ref }}$ & \\
\hline Tetanusschutzimpfung in den letzten 10 Jahren erhalten & \\
\hline Nein & $0,8(0,5-1,5)$ & $19,95(10,49-37,92)^{* *}$ & $19,46(16,23-37,02)^{* *}$ \\
\hline Ja & $14,3(13,1-15,6)$ & 19,4 &
\end{tabular}

\#gewichtete Angaben; *p-Wert $<0,05 ; * * \mathrm{p}-$ Wert $<0,001 ; \mathrm{KI}=$ Konfidenzintervall, $\mathrm{ABL}=$ Alte Bundesländer, $\mathrm{NBL}=\mathrm{Neue}$ Bundesländer; n.s.=nicht signifikant; Ref. = Referenzkategorie; Risikopopulation definiert als Zugehörigkeit zur Gruppe Medizinisches Personal, Personal in Gemeinschaftseinrichtungen, Frauen im gebärfähigen Alter ( $\leq 49$ Jahre); Personen mit im Haushalt lebenden Kindern $<1$ Jahr; ${ }^{t}$ bis 2005 war auch eine monovalente Impfung gegen Pertussis möglich, danach nur noch als Kombinationsimpfung mit Diphtherie- und Tetanuskomponente

\section{$\underline{\text { Krankenhaus-Apotheken-Survey }}$}

Das RKI erhielt von 133 (29,5\%) der 950 kontaktierten Krankenhausapotheken einen ausgefüllten Fragebogen zurück. Diese betreuten zwischen einem und 12 Krankenhäuser, insgesamt 454 (21,1\%) aller etwa 2.150 Krankenhäuser in Deutschland. Von den 454 Krankenhäusern lagen 60 (13,2\%) in den NBL; 326 (71,8\%) verfügten über eine Notaufnahme, 447 (98,5\%) betreuten Erwachsene und 182 (40,1\%) Kinder. Tabelle 3 zeigt die Anzahl der abgegebenen Impfdosen mit Tetanustoxoid-Komponentenimpfstoffen an Stationen und Notaufnahmen der Krankenhäuser. Die Mehrzahl der Impfdosen wurde als monovalente Tetanusimpfstoffe abgegeben; in Notaufnahmen $(76,3 \%)$ jedoch noch häufiger als auf Stationen $(68,0 \% ; \mathrm{p}<0.0001)$. Apotheken, die angegeben hatten ausschließlich Krankenhäuser zu beliefern, die keine Kinder betreuten (N=23 Apotheken; 60 Krankenhäuser), hatten einen noch höheren Anteil an monovalenten Tetanusimpfdosen an Notaufnahmen abgegeben $(82,6 \% ; p<0,0001)$. Auf Stationen wurde in diesen Krankenhäusern jedoch ein geringerer Anteil an monovalenten Impfdosen abgegeben (30,1\%, s. Tab. 3); allerdings beruht dies auf einem kleinen Teil aller abgegeben Impfdosen in diesen wenigen Kliniken (alle in den ABL). In Krankenhäusern aus den NBL wurden signifikant häufiger Tetanus-Kombinationsimpfstoffe eingesetzt als in den ABL (69,3\% vs. 17,7\%; $\mathrm{p}<0.0001)$. 
Tabelle 3

Abgegebene tetanushaltige Impfungen an von teilnehmenden Apotheken belieferte Krankenhäuser $(n=454)$

\begin{tabular}{|c|c|c|c|c|c|c|c|c|}
\hline \multicolumn{5}{|c|}{ Abgabe an Notaufnahmen } & \multicolumn{4}{|c|}{ Abgabe an Stationen } \\
\hline & \multicolumn{2}{|c|}{$\begin{array}{c}\text { von allen } \\
\text { Apotheken }(n=133)\end{array}$} & \multicolumn{2}{|c|}{$\begin{array}{l}\text { von Apotheken, die nur } \\
\text { Krankenhäuser für } \\
\text { Erwachsene betreuen } \\
(n=23)\end{array}$} & \multicolumn{2}{|c|}{$\begin{array}{c}\text { von allen } \\
\text { Apotheken }(n=133)\end{array}$} & \multicolumn{2}{|c|}{$\begin{array}{c}\text { von Apotheken, die nur } \\
\text { Krankenhäuser für } \\
\text { Erwachsene betreuen } \\
(n=23)\end{array}$} \\
\hline Impfstoff & Impfdosen & $\%$ & Impfdosen & $\%$ & Impfdosen & $\%$ & Impfdosen & $\%$ \\
\hline $\mathbf{T}$ & 141.173 & 76,3 & 19.741 & 82,6 & 15.182 & $68,7 *$ & 563 & 30,1 \\
\hline Td & 43.148 & 23,3 & 4.168 & 17,4 & 6.145 & $27,8 *$ & 1.249 & 66,7 \\
\hline TDaP & 175 & 0,1 & 0 & 0,0 & 334 & $1,5^{*}$ & 0 & 0,0 \\
\hline Tdap & 566 & 0,3 & 0 & 0,0 & 438 & $2,0^{*}$ & 60 & 3,2 \\
\hline Gesamt & 185.062 & 100,0 & 23.909 & 100,0 & 22.099 & 100,0 & 1.872 & 100,0 \\
\hline
\end{tabular}

*Unterschied Notaufnahmen vs. Stationen im Chi-Quadrat-Test signifikant: $\mathbf{p}<0,001$; TDaP: Tetanus-Diphtherie-azelluläre PertussisKombinationsimpfstoffe für Kinder $<5$ Jahren; Tdap: Tetanus-Diphtherie-azelluläre Pertussis-Kombinationsimpfstoffe mit reduziertem Diphtherie- und Pertussis-Antigengehalt für Kinder >4 Jahre und Erwachsene; Td: Tetanus-Diphtherie-Kombinationsimpfstoff; T: Monovalenter Tetanusimpfstoff 


\section{Diskussion und Schlussfolgerungen}

Seit 2009 wird von der STIKO die einmalige Impfung gegen Pertussis für alle Erwachsenen in Deutschland empfohlen. In der vorliegenden Studie werden erstmalig Daten zur Umsetzung der Pertussis-Impfempfehlung in zu Risikogruppen gehörenden Erwachsenen für die schon länger eine Impfempfehlung besteht - sowie in der erwachsenen Allgemeinbevölkerung vorgestellt.

Die Auswertung der GEDA-Daten zeigt, dass die Pertussis-Impfempfehlungen in Risikogruppen bisher völlig unzureichend umgesetzt wurden. Bemerkenswert ist, dass die Umsetzung der Empfehlung in den NBL besser als in den ABL (Pertussis-Impfquote: 19,2\% vs. 8.6\%), jedoch auch hier bei Weitem nicht ausreichend war. Fast zwanzig Jahre nach der Wiedervereinigung von Ost- und Westdeutschland bestehen hinsichtlich der Akzeptanz bestimmter Schutzimpfungen in der Bevölkerung offenbar immer noch deutliche Unterschiede zwischen den ABL und den NBL. Die höhere Akzeptanz der Pertussis-Impfung in den NBL, die auch für Tetanus- und Influenza-Impfungen beobachtet wurde [3,4,28], lässt sich möglicherweise als Folge der staatlich organisierten Impfprävention in der DDR verstehen. Der im Krankenhausapotheken-Survey dargestellte signifikante Unterschied bei der Verwendung von Tetanus-Kombinationsimpfstoffen zwischen NBL und ABL legt aber auch eine regional unterschiedliche Einstellung des Krankenhaus-Managements bzw. der Ärzteschaft nahe, die für die Umsetzbarkeit bzw. Umsetzung der Empfehlungen zuständig sind.

Insgesamt hatte im Jahr 2010 nur ein geringer Teil (5,9\%) der erwachsenen Allgemeinbevölkerung einen ausreichenden Impfschutz gegen Pertussis. Da die gewählte Impfstrategie vorsieht, die Pertussis-Impfung als Kombinationsimpfung mit der nächsten fälligen Td-Impfung zu verabreichen, wird es mindestens 10 Jahre dauern (Td-Impfschutz sollte alle 10 Jahre aufgefrischt werden), bis die STIKO-Empfehlung zur Pertussis-Impfung bei Erwachsenen theoretisch vollständig umgesetzt ist. Ausgehend von einer Tetanusimpfquote von $73 \%$ bei Erwachsenen in Deutschland [3], einer gleichmäßigen Verteilung der Tetanusimpfungen über die Jahre hinweg, sowie einer ausschließlichen Verwendung von Tdap-Kombinationsimpfstoffen zur Tetanusimpfung, wäre ein Anstieg der Pertussis-Impfquote unter Erwachsenen, die keiner Risikogruppe angehören, um ca. 7 Prozentpunkte pro Jahr zu erwarten. Personen in Risikogruppen sollten jedoch sofort - und nicht erst bei der nächsten fälligen Td-Impfung - geimpft werden; dies scheint den meisten Ärzten nicht klar zu sein. 
Die Ergebnisse des Krankenhaus-Apotheken-Surveys zeigen, dass die zum Studienzeitpunkt gültige Empfehlung der STIKO, eine indizierte Tetanusimpfung als Kombinationsimpfung mit Diphtherie-Komponente zu verabreichen, in deutschen Krankenhäusern unzureichend umgesetzt wurde. Insgesamt waren nur knapp ein Viertel der von Notaufnahmen bzw. knapp ein Drittel der von Stationen bestellten tetanushaltigen Impfstoffdosen die empfohlenen Kombinationsimpfstoffe. Eine Ausnahme bildeten hierbei Notaufnahmen in den NBL, bei denen tetanushaltige Kombinationsimpfstoffe einen deutlichen höheren Anteil (69,3\% versus 17,7\% in den ABL) ausmachten. Diese Ergebnisse lassen vermuten, dass auch zum heutigen Zeitpunkt die Compliance hinsichtlich der seit 2009 bestehenden Empfehlung zur Verwendung von Tdap-Kombinationsimpfstoffen im Rahmen der Tetanusprophylaxe allein schon mangels adäquater Impfstoffverfügbarkeit in den Notaufnahmen unzureichend sein könnte; ein aktueller Survey wäre wichtig, um diese Vermutung zu bestätigen. Als mögliche Erklärung für die mangelnde Umsetzung der Empfehlung waren 2007 noch Einschränkungen bezüglich der Durchführung von Impfungen für bestimmte Fachrichtungen zu sehen. Durch die Änderung der Musterweiterbildungsordnung der Bundesärztekammer im Jahr 2010, in der die Durchführung von Impfungen zu den allgemeinen Inhalten der Weiterbildung aufgenommen wurde, sind die Voraussetzungen für die Anwendung von Kombinationsimpfstoffen auch im Krankenhaussektor verbessert worden. Als weitere Gründe für eine bisher mangelhafte Umsetzung sind unzureichende Kenntnisse der STIKO-Empfehlung sowie die höheren Kosten für den etwas teureren Kombinationsimpfstoff denkbar. Es ist daher unabdingbar impfende Ärzte für die aktuellen STIKO-Empfehlungen zu sensibilisieren und Unsicherheiten zu beseitigen. Dies könnte beispielsweise durch Informationskampagnen in Krankenhäusern oder Publikationen in der Ärztepresse erfolgen. Der Einfluss solcher Maßnahmen auf die Umsetzung der STIKO-Empfehlung in Krankenhäusern sollte weiter verfolgt werden, z.B. durch eine Wiederholung des Krankenhaus-Apotheken-Surveys.

Zusammenfassend zeigen die vorliegenden Ergebnisse, dass offensichtlich große Wissenslücken bzw. Unsicherheiten zur Umsetzung der STIKO-Empfehlung sowohl bei niedergelassenen Ärzten (niedrige Impfquoten in Risikogruppen wie auch der erwachsenen Allgemeinbevölkerung) als auch bei Krankenhausärzten (geringe Verwendung von adäquaten Kombinationsimpfstoffen im Rahmen der postexpositionellen Tetanusprophylaxe) bestehen. Der hohe Anteil von Studienteilnehmern (GEDA10: 28\%), die nicht wussten, ob sie gegen Pertussis geimpft sind (Vergleich: Influenza: 0,2\%, Tetanus: 3,5\%), lässt zudem darauf 
schließen, dass der Bekanntheitsgrad der Pertussis-Impfung bzw. der entsprechenden STIKO-

\section{Schlussfolgerungen}

Vor dem Hintergrund der beträchtlichen Pertussis-Krankheitslast bei Erwachsenen in Deutschland und der hier gezeigten niedrigen Impfquoten gibt es ein großes Präventionspotential. Azelluläre Pertussis-Impfstoffe weisen eine lediglich moderate Impfeffektivität auf, die zudem deutlich mit der Zeit abnimmt [30]. Demgegenüber steht die hohe Kontagiosität des Erregers. Daher kann nur ein hoher Immunitätsgrad in der Gesamtbevölkerung zur nachhaltigen Eindämmung von Pertussis-Erkrankungen führen. Umso wichtiger ist es daher, neben Kindern und Jugendlichen auch Erwachsene gegen Pertussis zu impfen, vor allem im Hinblick darauf, dass Erwachsene am häufigsten die Infektionsquelle für Pertussis bei Säuglingen darstellen [15,16,26].

Alle Jugendlichen und Erwachsenen in Deutschland sollten auf die Notwendigkeit einer Impfung gegen Pertussis hingewiesen werden und den nächsten Arztkontakt nutzen, um ihren Impfstatus zu überprüfen. Insbesondere sollten alle Ärzte jeden Kontakt zu ihren Patienten zur 
Impfstatus-Kontrolle nutzen, um bestehende Impflücken zu schließen, und - sofern eine

\section{Danksagung}

Der Dank der Autoren gilt allen Mitarbeiter/innen des Robert Koch-Instituts, die an der Planung und Durchführung der GEDA-Surveys beteiligt waren, den Studienteilnehmern von GEDA09 und GEDA10, sowie dem ADAK und allen Apotheker/innen die an der Krankenhausapotheken-Befragung teilgenommen haben. 


\section{Literatur}

1. American Association for Public Opinion Research (AAPOR). Standard Definitions Final Dispositions of Case Codes and Outcome Rates for Surveys. 2011; verfügbar unter: http://www.aapor.org/AM/Template.cfm? Section=Standard Definitions $2 \&$ Template= /CM/ContentDisplay.cfm\&ContentID=3156 (Zugriffsdatum: 01/02/2012).

2. Beytout J, Launay O, Guiso N, et al. Safety of Tdap-IPV given one month after TdIPV booster in healthy young adults: a placebo-controlled trial. Human vaccines 2009; 5: $315-321$

3. Böhmer MM, Walter D, Krause G, et al. Determinants of tetanus and seasonal influenza vaccine uptake in adults living in Germany. Human vaccines 2011; 7: 13171325

4. Böhmer MM, Walter D, Müters S, et al. Seasonal influenza vaccine uptake in Germany 2007/2008 and 2008/2009: results from a national health update survey. Vaccine 2011; 29: 4492-4498

5. Centers for Disease Control and Prevention. Risk Factor Surveillance System: 2009 Summary Data Quality Report. 2010; verfügbar unter: ftp://ftp.cdc.gov/pub/Data/Brfss/2009_Summary_Data_Quality_Report.pdf (Zugriffsdatum: 01/01/2012).

6. David ST, Hemsley C, Pasquali PE, et al. Enhanced surveillance for vaccineassociated adverse events: dTap catch-up of high school students in Yukon. Can Commun Dis Rep 2005; 31: 117-126

7. De Serres G, Shadmani R, Duval B, et al. Morbidity of pertussis in adolescents and adults. The Journal of infectious diseases 2000; 182: 174-179

8. Greenberg DP, von Konig CH, Heininger U. Health burden of pertussis in infants and children. The Pediatric infectious disease journal 2005; 24: S39-43

9. Halperin SA, Sweet L, Baxendale D, et al. How soon after a prior tetanus-diphtheria vaccination can one give adult formulation tetanus-diphtheria-acellular pertussis vaccine? The Pediatric infectious disease journal 2006; 25: 195-200

10. Heininger U, Klich K, Stehr K, et al. Clinical findings in Bordetella pertussis infections: results of a prospective multicenter surveillance study. Pediatrics 1997; 100: E10

11. Heininger U, Stehr K, Christenson P, et al. Evidence of efficacy of the Lederle/Takeda acellular pertussis diphtheria and tetanus toxoids and pertussis vaccine but not the Lederle whole-cell component diphtheria and tetanus toxoids and pertussis vaccine against Bordetella parapertussis infection. Clin Infect Dis 1999; 28: 602-604

12. Robert Koch-Institut. Empfehlungen der Ständigen Impfkommission (STIKO) am Robert Koch-Institut / Stand: Juli 2012. Epidemiologisches Bulletin 2012; 30: 283-310

13. Kurth BM, Lange C, Kamtsiuris P, et al. Gesundheitsmonitoring am Robert KochInstitut. Sachstand und Perspektiven. Bundesgesundheitsblatt Gesundheitsforschung Gesundheitsschutz 2009; 52: 557-570

14. Lampert T, Kroll LE, Müters S, et al. Messung des sozioökonomischen Status in der Studie "Gesundheit in Deutschland aktuell" (GEDA). Bundesgesundheitsblatt 2013; 56: $131-143$

15. Lavine J, Broutin H, Harvill ET, et al. Imperfect vaccine-induced immunity and whooping cough transmission to infants. Vaccine 2010; 29: 11-16 
16. Lavine JS, Bjornstad ON, de Blasio BF, et al. Short-lived immunity against pertussis,

17. Postels-Multani S, Schmitt HJ, Wirsing von Konig CH, et al. Symptoms and complications of pertussis in adults. Infection 1995; 23: 139-142

18. Robert Koch-Institut. Impfempfehlungen der Ständigen Impfkommission (STIKO) am Rober Koch-Institut/Stand: Juli 2001. Epidemiologisches Bulletin 2001; 28: 203-215

19. Robert Koch-Institut. Empfehlungen der Ständigen Impfkommission (STIKO) am Robert Koch-Institut / Stand: Juli 2004. Epidemiologisches Bulletin 2004; 30: 235250

20. Robert Koch-Institut. Zusätzliche Pertussis-Impfung im Erwachsenenalter als TdapKombinationsimpfung bei der nächsten fälligen Td-Impfung - Empfehlung und Begründung. Epidemiologisches Bulletin 2009; 31: 299-311

21. Robert Koch-Institut. Keuchhusten-Erkrankungen in den neuen Bundesländern seit 2007. Epidemiologisches Bulletin 2010; 47: 463-471

22. Robert Koch-Institut. Daten und Fakten: Ergebnisse der Studie "Gesundheit in Deutschland aktuell 2010". Berlin: RKI; 2012

23. Rothstein E, Edwards K. Health burden of pertussis in adolescents and adults. The Pediatric infectious disease journal 2005; 24: S44-47

24. Stojanov S, Liese J, Belohradsky BH. Hospitalization and complications in children under 2 years of age with Bordetella pertussis infection. Infection 2000; 28: 106-110

25. von Konig $\mathrm{CH}$, Halperin S, Riffelmann M, et al. Pertussis of adults and infants. The Lancet infectious diseases 2002; 2: 744-750

26. Wendelboe AM, Njamkepo E, Bourillon A, et al. Transmission of Bordetella pertussis to young infants. The Pediatric infectious disease journal 2007; 26: 293-299

27. Wendelboe AM, Van Rie A, Salmaso S, et al. Duration of immunity against pertussis after natural infection or vaccination. The Pediatric infectious disease journal 2005; 24: S58-61

28. Wiese-Posselt M, Leitmeyer $\mathrm{K}$, Hamouda $\mathrm{O}$, et al. Influenza vaccination coverage in adults belonging to defined target groups, Germany, 2003/2004. Vaccine 2006; 24: 2560-2566

29. World Health Oranization. Pertussis vaccines: WHO position paper. Wkly Epidemiol Rec 2010; 85: 385-400

30. Zhang L, Prietsch SO, Axelsson I, et al. Acellular vaccines for preventing whooping cough in children. Cochrane database of systematic reviews (Online) 2012; 3 : CD001478

31. Zhang X, Rodriguez ME, Harvill ET. O antigen allows B. parapertussis to evade B. pertussis vaccine-induced immunity by blocking binding and functions of crossreactive antibodies. PloS one 2009; 4: e6989 


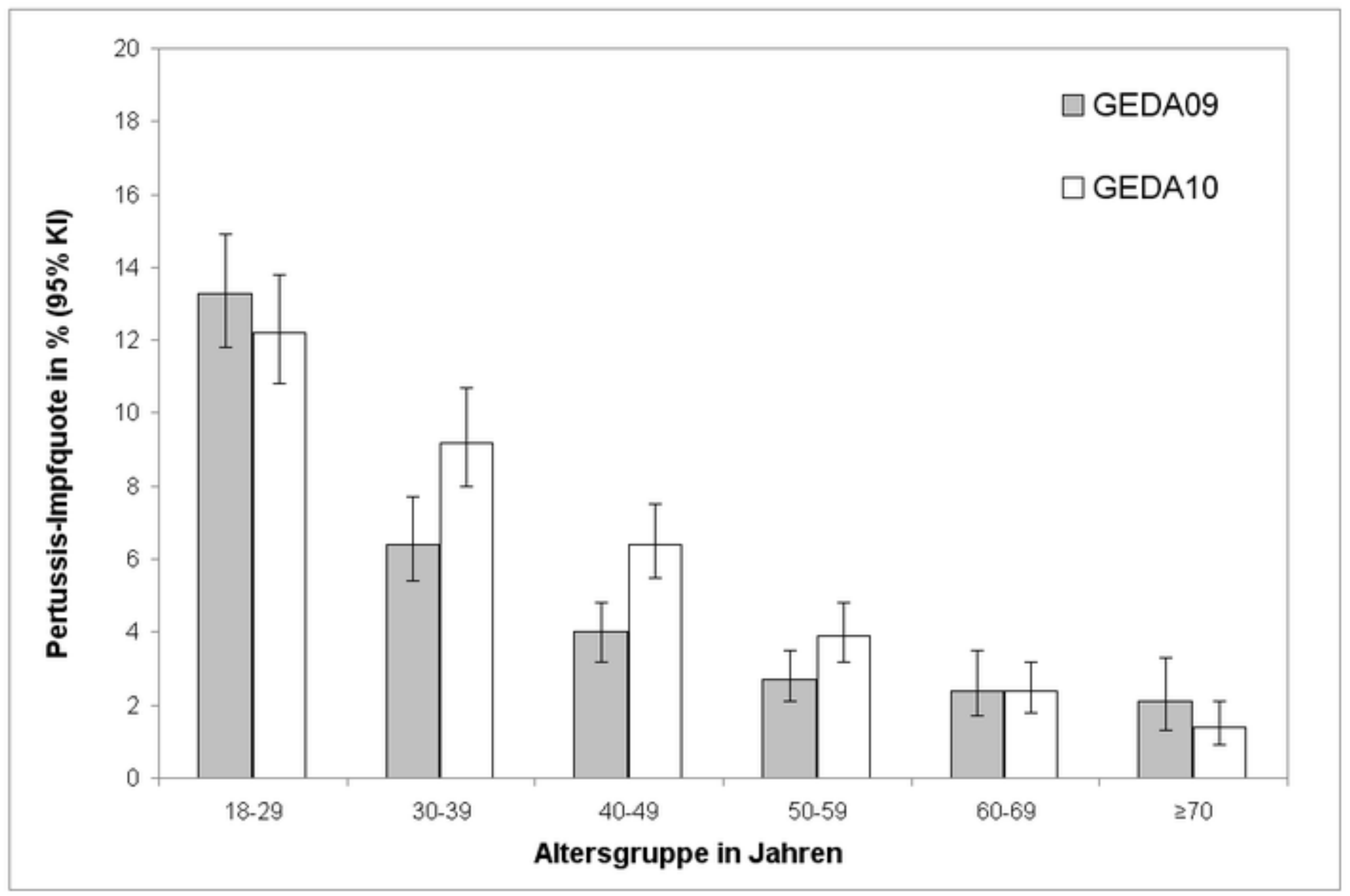

